POPULAR SUMMARY

Assimilation Experiments of One-dimensional Variational Analyses

with GPS/MET Refractivity

Paul Poli ${ }^{1,3 *}$, Joanna Joiner ${ }^{2}$

${ }^{1}$ Joint Center for Earth Systems Technology, Baltimore, Maryland, USA

${ }^{2}$ Laboratory for Atmos oheres, NASA Goddard Space Flight Center, Greenbelt, Maryland, USA

${ }^{3}$ also at Météo-France, Toulouse, France

The Global Positioning System enables positioning in 3 dimensions about our planet. The signals sent by twenty-four satellites travel through our atmosphere down to the small receivers used by the civilian community and the military. These signals are electromagnetic waves; they do not travel in a straight line because of varying meteorological conditions. Instead, they bend towards the surface and ravel slower than in a vacuum.

A small GPS receiver was launched in the satellite GPS/MET in 1995. It became possible to perform radio occultations around the Earth: the source - one of the 24 GPS satellites - is seen by the receiver as it rises or sets around the other side of the Earth. When the source disappears, the receiver r,rogressively loses the signals. The delay induced by the atmosphere can be infered by measu ing measuring accurately the time delay between the emission and the reception of the signel.

In a previous study we had shown one method to combine GPS/MET data with numerical weather forecasts in a statiscally optimal way to yield improved profiles of temperature.

We use these observations and assimilate them in the Data Assimilation Office Finite Volume Data Assimilation System (FVDAS). The information brought by the temperature profiles is used by the FVDAS to perform global three dimensional weather analyses of the atmosphere. We evaluate the impact of the GPS/MET data on the global analyses and the weather forecasts.

"Corresponding author address: Paul Poli, Code 910.3, NASA/GSFC, Greenbelt, MD 20771. e-mail: ppoli@dao.gsfc.nasa.gov 



\section{Assimilation Experiments of One-dimensional Variational Analyses with GPS/MET Refractivity}

Paul Poli ${ }^{1,3}$ and J ranna Joiner ${ }^{2}$

1 Joint Center for Earth Systems Technology (JCET), University of Maryland Baltimore County Baltimore, MD 21250 USA ppoli@dao.gsfc.nasa.gav

2 NASA Laboratory for Atmospheres, Goddard Space Flight Center, Greenbelt, MD 20771 USA jjoiner@dao.gsfc.nasa.gov

3 also at Météo France Centre National de Recherches Météorologiques, Toulouse, France

\section{Introduction}

The GPS/MET 1995 experiment demonstrated the feasability of remote soundings of the Exrth's atmosphere using the GPS radio occultation technique [5]. Subsequent studies assessed the information content of such measurements (e.g. [3]) Several groups have attempted to assimilate the GPS radio occultation mersurements into a global Data Assimilation System (DAS). These attempts followed in general the methodology proposed by Eyre [2], and range from disect four-dimensional variational assimilation of bending angles to assimilation of inverted profiles of temperature and/or humidity. We present here an hybrid approach. We attempted to take advantage of the accurracy of curren', weather models to constrain one-dimensional variational analyses using GPS refractivity. Retrieved profiles of atmospheric parameters were then assimilated like other types of observations in a global DAS for issuing the next fore ast period.

\section{One-dimensional Variational (1DVAR) Analyses with Refractivity}

The application of one-dimensional variational analysis (1DVAR) to the retrieval of geophysical parameters from GPS radio occultation has already been discussed by various authors (e.g. [3], [9]). We briefly describe the main ideas. Technical details can be found in [10].

\subsection{DVAR Analysis Theory}

Air refractivity $N$ for GPS signals frequencies ( $L$-band) can be written (neglecting scattering $i$ ind ionospheric contribution)

$$
N=b_{1} \frac{P}{T}+b_{2} \frac{P_{w}}{T^{2}}
$$


[11], where $P$ is the air pressure in $h \mathrm{~Pa}, T$ the temperature in $\mathrm{K}, P_{w}$ the water vapor partial pressure in $\mathrm{hPa}, b_{1}=77.6 \mathrm{~N}$-unit. $\mathrm{K} \cdot \mathrm{hPa}{ }^{-1}$, and

$b_{2}=3.73 \times 10^{5} \mathrm{~N}$-unit $\cdot \mathrm{K}^{2} \cdot \mathrm{hPa}^{-1}$. It is obvious from equation (1) that the direct inversion of refractivity measurement into temperature, humidity and pressure is not possible without assumptions. One way to address the temperature/humidity ambiguity is to use physical constraints via a IDVAR approach. A priori information (or background) is combined with GPS refractivity observations in a statistically optimal way to yield analyzed profiles and temperature and humidity. An important hypothesis is that all errors are supposed to be non-biased and normally distributed.

\subsection{GPS/MET Observations}

We used 797 occultations collected during 'Prime Time 2' of the GPS/MET 1995 experiment, when the GPS Anti-Spoofing encryption had been turned off from June 21st until July 4th, 1995. This, along with changes in the software of the receiver enabled tracking of the GPS occultations deep in the troposphere. In order to use these obervations in the IDVAR scheme we assumed the following standard deviation error in refractivity: $1 \%$ below $5 \mathrm{~km}$ altitude, $0.2 \%$ between $5 \mathrm{~km}$ and $30 \mathrm{~km}$ altitude. We discarded the refractivity observations above $30 \mathrm{~km}$ altitude.

\subsection{Background Information}

The Data Assimilation Office (DAO) Finite Volume Data Assimilation System (FVDAS) [1] provided the 6-hour forecasts used as a background in the IDVAR implementation. The forecasts were bilinearly interpolated from $2 \times 2.5$ degrees grid to the location of the GPS occultation. No time interpolation was performed. The forecast fields (from the surface to the $0.01 \mathrm{hPa}$ level) were interpolated to 46 levels for the IDVAR analysis.

\subsection{GPS 1DVAR Results}

Due to a positive bias in the background minus observation refractivity the analyzed IDVAR humidity profiles presented systematic departure from the original 6-hour forecast used to initiate the retrieval. These departures were inconsistent with humidity measurements reported by nearby radiosondes [10]. We hence decided to investigate this subject further before assimilating these analyzed humidity profiles into the FVDAS.

Figure 1 shows temperature comparisons with nearby RS (less than $280 \mathrm{~km},+/-3$ hours). The 1DVAR analysis has smaller bias than the background above the $200 \mathrm{hPa}$ level. The standard deviation of the background is larger than the 1DVAR analysis between $850 \mathrm{hPa}$ and $20 \mathrm{hPa}$. This suggests that the IDVAR analyzed temperature profiles may provide useful information to a global Data Assimilation System (DAS). 


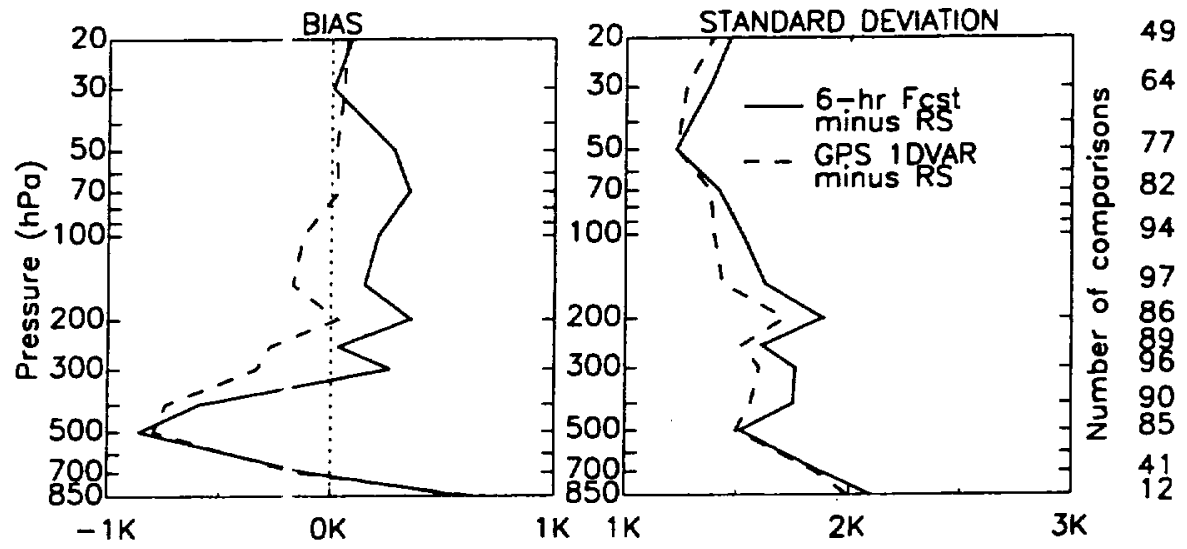

Fig. 1. Temperatur comparisons of 6 -hour forecasts and GPS $1 D V A R$ with nearby radiosondes; numbel of comparisons on the right-hand side

\section{Assimilation of GPS 1DVAR Profiles in the Finite Volume Data Assimilation System (FVDAS)}

\subsection{Description of the FVDAS and Link with GPS IDVAR Analysis}

The FVDAS relies on a NASA/NCAR (National Aeronautics and Space Administration/National Center for Atmospheric Research) Finite Volume General Circulation Mc.del (GCM). The GCM dynamical core uses a Lagrangian vertical coordinate system [7]. The assimilation part of the FVDAS is a Physical-space Sta istical Analysis System (PSAS) [1]. The FVDAS assimilates conventional , bservations such as radiosondes (RS) as well as 1DVAR cloud-cleared retric ved profiles of temperature and humidity derived from TIROS Operationel Vertical Sounder (TOVS) level-1b brightness temperatures [4].

When running he FVDAS from June 21st, 1995 to July 4th, 1995, the latest available fore ast was used to perform the GPS IDVAR analysis. Then, the GPS IDVAR a nalyzed temperature profiles were assimilated in the FVDAS using PSAS. They were assigned the same error characteristics as radiosondes. Due to significant refractivity differences between 6-hour forecast background and olservation [10], we chose to restrict the assimilation of 1DVAR retrievals to the region $500 \mathrm{hPa}-30 \mathrm{hPa}$ in the $\left[30^{\circ} \mathrm{S}, 30^{\circ} \mathrm{N}\right]$ latitude band. At other latitudes, 1DVAR retrievals were assimilated between the surface and the $30 \mathrm{nPa}$ level. We refer to this experiment as 'GPS'.

In order to eval late the impact of the GPS 1DVAR analyses on the FVDAS we also performed a 'CONTROL' experiment with no GPS data. 


\subsection{Impact on Analyses}

Without any other observation other than those assimilated in the two experiments, it is difficult to determine which analysis is the closest to the atmospheric "truth". Figure 2 shows the difference between an analysis with GPS data minus the corresponding 'CONTROL' analysis for 1995-06-29 at 00GMT. In this example we used the same first guess for perform both analyses. Consequently, the differences we see are solely due to the GPS temperature observations assimilated at 00GMT on 1995-06-29. The differences in the analyzed winds enables to assess how the GPS temperature observations changed the atmospheric circulation through the mass-wind balance. For example, negative temperature increments corresponding to negative geopotential heights increments induced cyclonic-type increments in winds, i.e. horizontal circulation differences oriented clockwise in the Southern hemisphere.

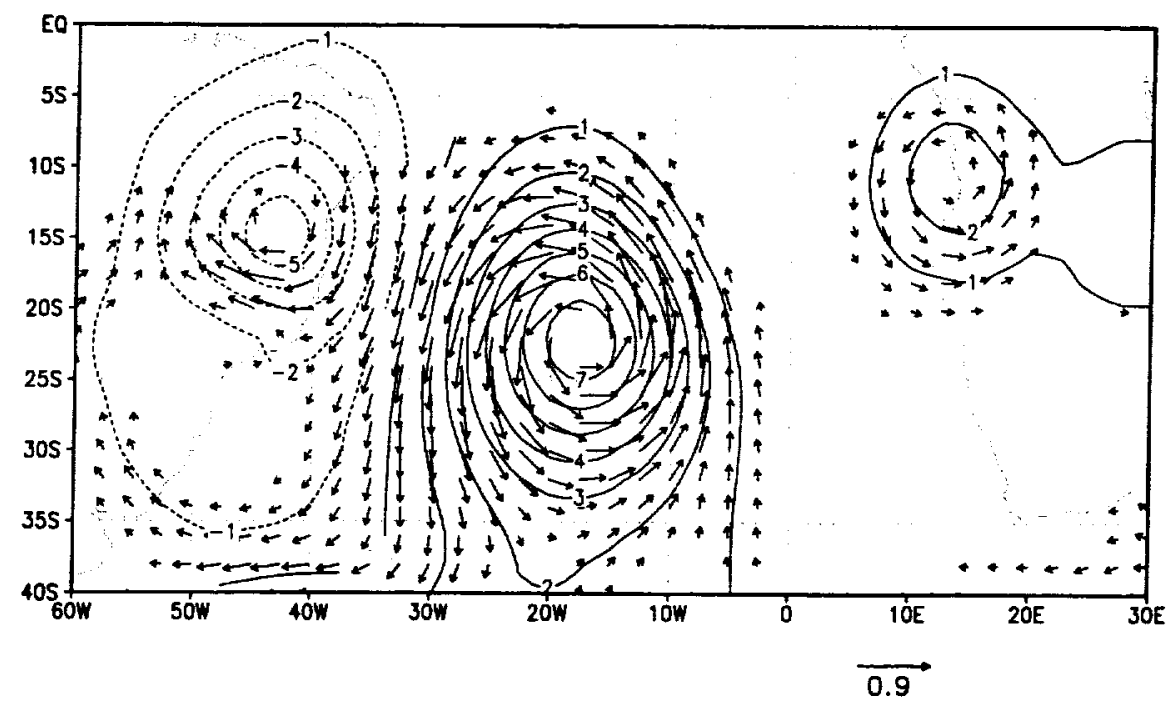

Fig. 2. Difference 'GPS' analysis minus 'CONTROL' at $250 \mathrm{hPa}$. Contours designate geopotential heights, arrows represent wind.

\subsection{Impact on 6-hour Forecasts}

We compared the 6-hour forecasts from the two experiments with radiosonde observations. These observations may be considered as an independent validation. Figure 3 shows zonal averages of the standard deviation of the observation minus forecast for the meridional wind. The 'CONTROL' has larger 
errors in the Soutlern hemisphere than in the Northern hemisphere where it is more constraine 1 by in situ observations. The shaded areas in the 'GPS' minus 'CONTROI' indicate reduced errors in the 'GPS' 6-hour forecast as compared to the 'CONTROL'. Differences are more apparent in the Southern hemisphere.
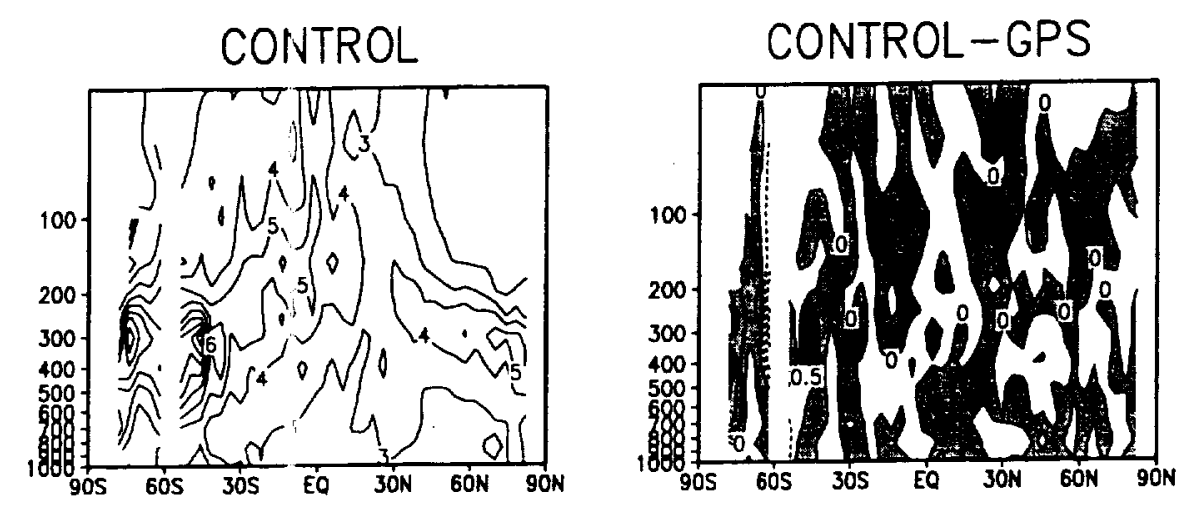

Fig. 3. Radiosonde sbservations minus 6-hour forecast standard deviation error for meridional wind. Ne rative coutours (dotted lines) in the 'CONTROL' minus 'GPS' suggest a negative in nact of the GPS data; Shaded areas indicate a slight positive impact.

\subsection{Impact on 5 day Forecasts}

We ran a series of $\leftleftarrows$-day forecasts from daily 00GMT analyses from June 21st until July 4 th, 199 . Forecast scores were computed in the form of Root Mean Square (RMS) and anomaly correlation for sea level pressure and $500 \mathrm{hPa}$ geopotential height. We used three different verifying analyses: (a) own analysis for each experiment, (b) 'CONTROL' analyses, and (c) European Centre for Medium-range Weather Forecasts (ECMWF) operational 1995 analyses. The GPS data induced slight differences on forecast scores of individual forecasts. The average of the 14 forecasts showed very little difference between 'CONTROL' and 'GPS'. The small number of forecasts issued and the limited amount of GF'S data assimilated does not enable us to fully assess the impact of GPS refi activity data in a global data assimilation system.

\section{Conclusions and Future Work}

We have implement ed a 1DVAR scheme to analyze temperature and humidity profiles using GPS radio occultation refractivity. This scheme was incorporated in the assimilation cycle of the FVDAS and we performed assimilation 
experiments using GPS/MET refractivity. We found mixed results in terms of impact of the GPS data on forecasts. Clearly, the limited amount of GPS observations limits the significance of the present work and more GPS data are needed to assess their impact on a global data assimilation system.

Even in a 1DVAR framework with limiting hypotheses such as no account of the atmospheric horizontal structure, we found a positive impact of GPS refractivity on the analysis of temperature. However, the humidity retrievals were not satisfactory partly because of a "refractivity bias". The source of this bias observed also in the Champ radio occultation measurements [8] has to be understood, because it plays even a more critical role in a direct assimilation where analysis increments cannot be separated. Similar assimilation experiments will be performed using the Champ and SAC-C GPS radio occultation observations, and the refractivity bias will be investigated.

\section{References}

1. Data Assimilation Office (DAO) (2000): Algorithm Theoretical Basis Document (ATBD) version 2, Draft. NASA Goddard Space Flight Center, Greenbelt, Maryland, USA

2. Eyre, J.R. (1994): Assimilation of radio occultation measurements into a numerical weather prediction system. ECMWF Tech. Memo., 199, Reading, UK

3. Healy, S.B., Eyre, J.R. (2000): Retrieving temperature, water vapour and surface pressure information from refractive index profiles derived by radio occultation: a simulation study. Quart. J. Roy. Met. Soc., 126, 1661-1683

4. Joiner, J., Rokke, L. (2000): Variational cloud-clearing with TOVS data. Quart. J. Roy. Met. Soc., 126, 725-748

5. Kursinski, E.R., Hajj, G.A., Bertiger, W.I., Leroy, S.S., Meehan, T.K., Romans, L.J., Schofield, J.T., McCleese, D.J., Melbourne, W.G., Thornton, C.L., Yunck, T.P., Eyre, J.R., Nagatani, R.N. (1996): Initial results of radio occultation observations of Earth's atmosphere using the global positioning system. Science, 271, 1107-1110

6. Kursinski, E.R., Hajj, G.A., Schofield, J.T., Linfield, R.P., Hardy, K.R. (1997): Observing Earth's atmosphere with radio occultation measurements using the global positioning system. J. Geophys. Res., 102, 23,429-23,465

7. Lin, S.J. (1997): A finite-volume integration method for computing pressure gradient force in general vertical coordinates. Quart. J. Roy. Met. Soc., 123, 1749-1762

8. Marquardt, C., et al. (2002): Status of CHAMP radio occultation products and their validation. Talk at the First CHAMP Science Meeting, Potsdam, Germany

9. Palmer, P.I., Barnett, J.J., Eyre, J.R., Healy, S.B. (2000): A non-linear optimal estimation inverse method for radio occultation measurements of temperature, humidity and surface pressure. J. Geophys. Res., 105, 17,513-17,526

10. Poli, P., Joiner, J., Kursinski, E.R. (2001): 1DVAR analysis of temperature and humidity using GPS radio occultation refractivity data. Submitted to J. Geophys. Res.

11. Smith, E.K., Weintraub, S. (1953): The constants in the equation for atmospheric index at radio frequencies. Proc. IRE, 41, 1035-1037 